\title{
Spectral Analysis of Instantaneous Frequency Responses to Sinusoidal Stimulation in Cutaneous Mechanoreceptor Afferent Units of Frogs
}

\author{
Kohachi Taniguchi and Hisashi Ogawa \\ Department of Physiology, Kumamoto University Medical School, \\ Kumamoto, 860 Japan
}

\begin{abstract}
While applying the Fast Fourier Transform to the instantaneous frequency responses to sinusoidal indentations of the cutaneous mechanoreceptor afferent units in frogs, we examined quantitatively the dynamic responses of these units. In two kinds of slowly adapting (SA) units, i.e., frog type I and frog type II units, and in rapidly adapting (RA) type I units, the instantaneous frequency responses could be reconstructed by summation of the DC component and the major 3-5 harmonics in the power spectra. In both types of SA units, the fundamental wave was the largest in power of the spectrum, but in the RA units, the 2nd harmonic was the largest. In SA units, the phase of the fundamental wave advanced by $20-55^{\circ}$ relative to the sinusoidal stimulation, but the phase of the 2 nd harmonic of the RA units advanced by ca. $90^{\circ}$. The magnitude of each component in the power spectra, especially the fundamental wave of the two SA units and the 2nd harmonics of the RA unit, increased with an increase in stimulus amplitude and frequency. The phases of the harmonics in both SA and RA types were fairly constant over varying amplitudes and frequencies of sinusoidal stimulation. The present findings indicate that both the frog type I and type II cutaneous mechanoreceptor afferent units detect both indentation magnitude and positive velocity, and that the RA type I cutaneous mechanoreceptor afferent units detect the stimulus velocity.
\end{abstract}

Key words: cutaneous mechanoreceptor, sinusoidal indentation, instantaneous frequency, Fast Fourier Transform, frog.

Afferent fibers innervating cutaneous mechanoreceptors are classified into several subtypes, based on properties of the response to mechanical stimuli, such as "ramp and plateau" or sinusoidal indentation of the skin. Slowly-adapting (SA)

Received for publication November 2, 1987 
fibers are thought to carry the signal related to the magnitude of skin indentation, and rapidly-adapting (RA) fibers are considered to detect the speed of deformation. The fibers innervating the Pacinian corpuscle are regarded as the detector of stimulus acceleration (Burgess and PERL, 1973; IGGo 1974). However, the basis for this classification seems rather vague (cf. GiBson et al. 1975).

Sinusoidal indentation of the skin contains three known components of mechanical stimulation: displacement, velocity, and acceleration. It has been shown that each type of mechanoreceptor responds to sinusoidal skin indentations, with a specific threshold response phase (CREMERS, 1972). In a preceding paper, we examined threshold response phases of four types of cutaneous mechanoreceptor afferents in frogs (two types of SA units, i.e., Ft I and Ft II units; and two types of RA units, i.e., RA type I and RA type II units) by measuring phases of their first spikes discharged in each cycle of successive sinusoidal skin indentations (TANIGUCHI et al., 1984). Both Ft II units and RA type II units followed expectation as displacement or acceleration detectors, as was suggested from the experiment using "ramp and plateau" indentation (OGAwA et al., 1981). On the other hand, neither Ft I nor RA type I units conformed to the theoretical expectation as displacement or velocity detectors, a finding inconsistent with results of previous experiments using "ramp and plateau" indentation (OGAWA et al., 1981). The deviation from the expectation of the average threshold response phase of the latter units could be attributed to the nature of the mechanoreceptors. The mechanoreceptor afferent unit may encode two or more components of mechanical indentation, and the threshold response phase method is not sensitive enough to separate the two components, or mechanoreceptors may become habituated to repetitive stimuli and the threshold phase may be gradually delayed.

Spectrum analysis has been utilized to analyze properties of physical or biological systems which respond to a stimulus consisting of several sinusoidal waves with different frequencies and phases (FrENCH et al., 1972; XERRI et al., 1987). Therefore, this approach is suitable for analyzing the coding mechanism of cutaneous mechanoreceptor afferent units, when a discrete form of their responses can be transformed into an analogue wave to apply spectrum analysis (SAYERS, 1973; Charnock and ManeniCa, 1978).

We quantitatively examined dynamic responses of the cutaneous mechanoreceptor afferent units of frogs, the objective being to investigate the related coding mechanisms. Power spectra of the instantaneous frequency responses (IFRs) of Ft I, Ft II, and RA type I units were calculated by applying a Fast Fourier Transform (FFT). A preliminary report has been published in abstract form (TANIGUCHI and OGAWA, 1986).

\section{METHODS}

Single afferent units were recorded from the sciatic nerve innervating cutaneous mechanoreceptors on the plantar surface of the hind limb of the American bullfrog, 
Rana catesbeiana. The recording procedures were as described (TANIGUCHI et al., 1984).

Mechanical stimulation. Sinusoidal indentations (frequency, 0.1 to $10 \mathrm{~Hz}, 1 / 2$ peak-to-peak amplitude, $200 \mu \mathrm{m}$ in the non-warty skin and $800 \mu \mathrm{m}$ in warty skin) were applied with a moving coil transducer (MSE 101 vibrator, Ling, Hertfordshire, England; OGawa et al., 1981) to receptive field on the skin. To avoid tapping of the skin with the probe, a steady indentation of 250 or $1,000 \mu \mathrm{m}$ (in the non-warty or warty skin, respectively) was given as a bias. Movements of the stimulus probe were monitored by a mechano-electric transducer (7DCDT, Hewlett Packard, Massachusetts, U.S.A.). Impulse discharges of single mechanoreceptor afferent units and outputs of mechano-electric transducer were simultaneously recorded on FM instrumentation tape for subsequent off-line data analysis. To measure the conduction time from the receptive field to the recording site, electrical stimuli $(0.2 \mathrm{~ms}$ twice the threshold voltage) were delivered to the nerve terminals by a pair of needle electrodes inserted into the skin.

Data processing. The responses obtained during the first 32 successive cycles of sinusoidal indentations were studied. With the aid of a microcomputer (7T17, NEC San-ei Instrument, Tokyo, Japan), post-stimulus time intervals of impulse sequences were measured with a time resolution of $0.1 \mathrm{~ms}$ after the time point when the output of the mechano-electric transducer crossed the neutral position from withdrawal to the indentation phase, i.e., a positve-going zero-crossing time point. The conduction time of the nerve fiber was then subtracted from the observed poststimulus time intervals.

To convert spike trains to an analogue waveform, there are at least two methods, i.e., the IFR and the method using counts of spikes per time bin. In the present study, we used IFR, a series of the reciprocals of interspike intervals (ISIs) assigned at the time periods of corresponding ISI, since neutral information is considered to reside in variation of length of ISIs (BULLOCK, 1967). IFRs to sinusoidal stimulation were obtained as follows (Fig. 1): firstly, sequences of ISIs were obtained by measuring ISIs on the basis of spike generation time points $\left(t_{m-1}\right.$, $\left.t_{m}, t_{m+1}, t_{m+2}, \cdots, t_{m+n}\right)$ referring to the positive-going zero-crossing time point. An IFR between the two spikes was then calculated by means of a reciprocal of their ISI; e.g., IFR at ISI between the $(m-1)$ th and the $m$ th spikes is expressed by $1 /\left(t_{m}-t_{m-1}\right)$, and referred to level I in Fig. 1Ac. A peak of the IFR in a single trial exists at the shortest ISI (e.g., level II at an ISI between $t_{m}$ and $t_{m+1}$ in Fig. 1A), but not at a time point of any spike. A peak of the average IFR is at the time interval where a unit under study produced the shortest ISI in most trials (Fig. 1B). When the unit produces phase-locked discharges, the average IFR and all of the IFRs obtained in single trials share the peak at the same time interval. Thus, the IFR data were transformed into a equidistant time series data of 512 per cycle, after LUCZAK and LAURIG (1973).

Since the first cycle of the sinusoidal indentation started from varying phases and contained high components of stimulation, we omitted from the data analysis 

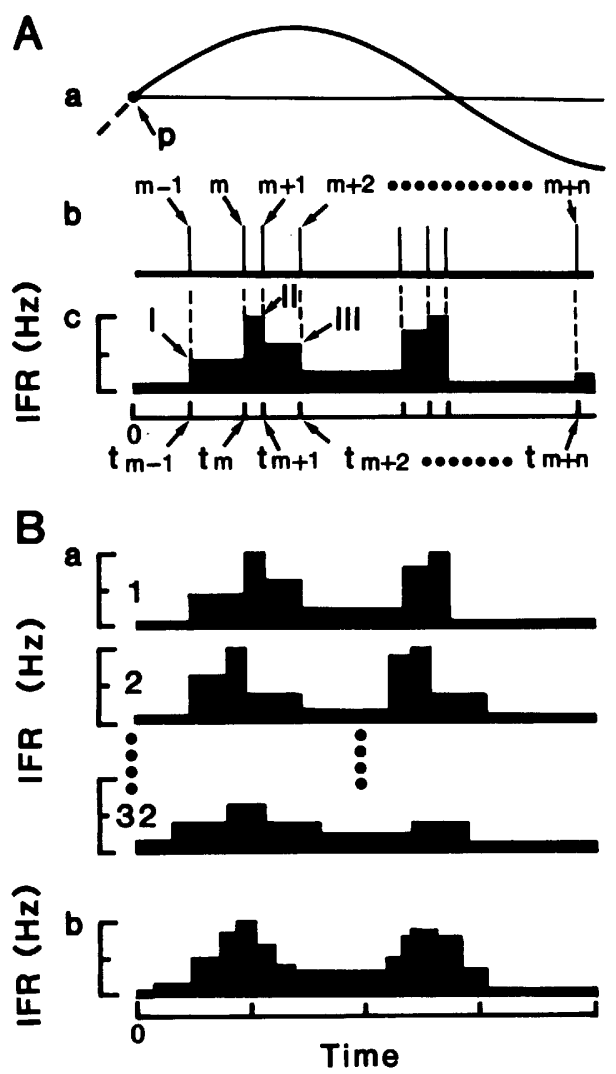

Fig. 1. Scheme illustrating the method of obtaining an average instantaneous frequency response (IFR) from a sequence of interspike intervals (ISIs). A, transformation of sequence of ISIs into IFR: a, stimulus waveform; $p$ indicates positivegoing zero-crossing time points; $\mathrm{b}$, spike train $(m-1, m, m+1, m+2, \cdots, m+n)$ in a trial; c, IFR obtained from reciprocals of ISIs. Times of spike generations $\left(t_{m-1}\right.$, $\left.t_{m}, t_{m+1}, t_{m+2}, \cdots, t_{m+n}\right)$ were measured referring to the zero-crossing time point. Sequences of ISIs were made by assessing differences between the consecutive spike generation times, e.g., $\left(t_{m}-t_{m-1}\right),\left(t_{m+1}-t_{m}\right),\left(t_{m+2}-t_{m+1}\right), \cdots$. The magnitudes of I, II, and III values in the IFR represent $1 /\left(t_{m}-t_{m-1}\right), 1 /\left(t_{m+1}-t_{m}\right)$, and $1 /\left(t_{m+2}-t_{m+1}\right)$, respectively. Each IFR is assigned to the ISI, by use of which the value is obtained. Note that the shortest ISI, ISI of $\left(t_{m+1}-t_{m}\right)$, produces the largest IFR, level II in the figure. B, average of IFRs over 32 trials: a, IFRs $1,2, \cdots, 32$ obtained from ISIs in trials $1,2, \cdots, 32$; b, average IFR over 32 trials.

the series of impulses in the 1st cycle. Average cycle time IFRs over the 31 cycles were calculated. In a part of the present study, the remaining 31 successive cycles were separated into three sections, the first ten cycles (2nd-11th), the second ten (12th-21st), and the third ten (22nd-31st). Then, the average cycle time IFRs were similarly obtained for each of the three sections over the 30 cycles. With the aid of 
the Signal Processer and a desktop microcomputer (HP-85, Hewlett-Packard, Oregon, U.S.A.), we applied a Fast Fourier Transform (FFT; CoOLEY and TUKEY, $1965)$ to the average cycle time IFRs.

It is generally accepted that the power is significant when it is larger than $1 / \sqrt{N}$ times the maximum power, where $N$ indicates the number of trials or the number of averages (BENDAT and Piersol, 1980), i.e., $N=31$ for most cases in the present study. However, when the power of a certain harmonic was equal to or greater than $10 \%$ of the maximum power, except for that of the DC component, we considered the power to be significant for convenience in the present study. Thus, we took into consideration a larger than generally expected number of components in the power spectrum.

\section{RESULTS}

A total of 19 single units were isolated: 6 were classified as Ft I units, 3 as Ft II units, 4 as RA type I units, and 6 as RA type II units, on the basis of response patterns to "ramp and plateau" indentation and on the basis of tuning curves to sinusoidal indentation (OGAWA et al., 1981, TANiguchi et al., 1984). The Ft I, RA type I, and RA type II units had receptive fields on the warty skin, and the Ft II units had receptive fields on the non-warty skin. Of these four types, we chose Ft I, Ft II, and RA type I units, for the present study, because they discharged vigorously enough in a cycle to produce relatively smooth IFRs, a prerequisite for FFT application. When the stimulus probe was set 250 or $1,000 \mu \mathrm{m}$ below the resting surface of the skin, all SA units of either type produced a large number of discharges in the first several seconds but ceased firing within a few minutes. Thus in the case of the SA units, we recorded the spike discharges after they had adapted to the steady bias displacement (see METHODS).

\section{General features of the spike discharge phase and IFR to sinusoidal stimulation}

Figure 2 shows the average IFRs of three mechanoreceptor afferent units over 32 cycles of standard sinusoidal indentations. Both Ft I and Ft II units showed impulse discharges at the phase of increasing indentation. The average number of impulses per cycle gradually decreased with repetition: latencies of the first spikes gradually became longer and the duration of the discharges became shorter (Fig. $2 \mathrm{Ab}, \mathrm{Bb}$ ). This tendency was more marked in Ft I (Fig. 2Ab) than in Ft II units (Fig. 2Bb). The cycle time IFR showed a triangular shape in the Ft I units (Fig. 2Ac), and a bell shape in the Ft II units (Fig. 2Bc). These features were observed in all of the six Ft I and the three Ft II units studied.

On the other hand, the discharge pattern of RA type I units differed from that of the SA units. Phase-locked spikes were evoked near the two zero-crossing time points, i.e., the positive-going zero-crossing time point and the negative-going zerocrossing time points, i.e., the transition from the indentation to withdrawal phase, and vice versa. Thus, the RA type I units showed a cycle time IFR with two peaks 


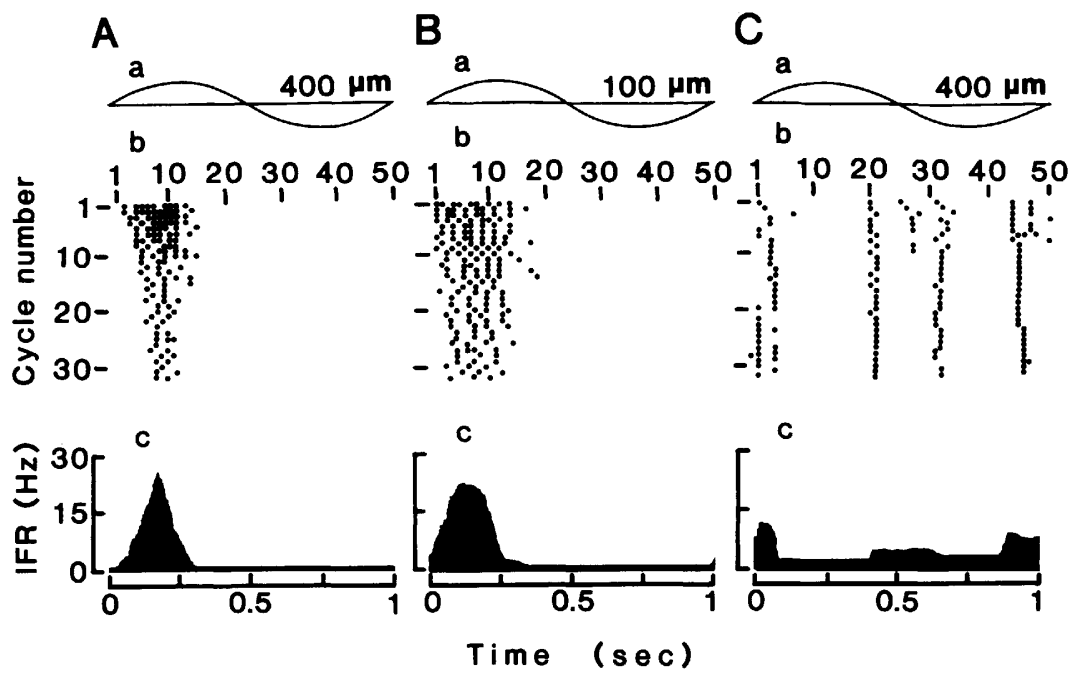

Fig. 2. Impulse discharge pattern and instantaneous frequency response of mechanoreceptor afferents to $1 \mathrm{~Hz}$ sinusoidal indentation. A: frog type I (Ft I) unit. B: frog type II (Ft II) unit. C: RA type I unit. a: trace of sinusoidal stimulation. The upward direction indicates indentation; numerals indicate amplitude of sinusoidal indentations. b: occurrence of spikes, displayed by dots at a corresponding bin, while one sinusoidal cycle was divided into 50 bins. c: cycle time IFRs shown by means of reciprocals of interspike intervals during the 32 successive cycles of sinusoidal indentation.

(Fig. 2Cc), in contrast to a single peak cycle time IFR in SA units. Out of the four units examined, the unit of Fig. IC produced a larger IFR at the positive-going zero-crossing time point than at the negative-going zero-crossing time point. In another unit, the IFR at the negative-going zero-crossing time point was much larger than at a positive-going zero-crossing time point. In the remaining two units, the IFR showed almost the same magnitude at both positive-going and negativegoing zero-crossing time points.

Spectral analysis of the cycle time IFR for a total of 31 cycles

Power spectrum. Three units were randomly selected from each population of Ft I, Ft II, and RA type I units, and FFT was applied to their average cycle time IFRs made over the 31 cycles from the 2nd to 32nd cycles. Examples of power spectra thus obtained are shown in Fig. 3. In all SA units of either type (Fig. 3A and $B)$, the power of the fundamental wave was the largest in the spectrum, and the power of the harmonics became smaller, with increased order. The power of the harmonics of higher than 5 th orders was less than $10 \%$ of the power of the largest component, except for the DC component.

The power of the 2 nd harmonic was the largest in the spectrum of all the RA type I units examined (Fig. 3C to E), except for the DC component, though this was 

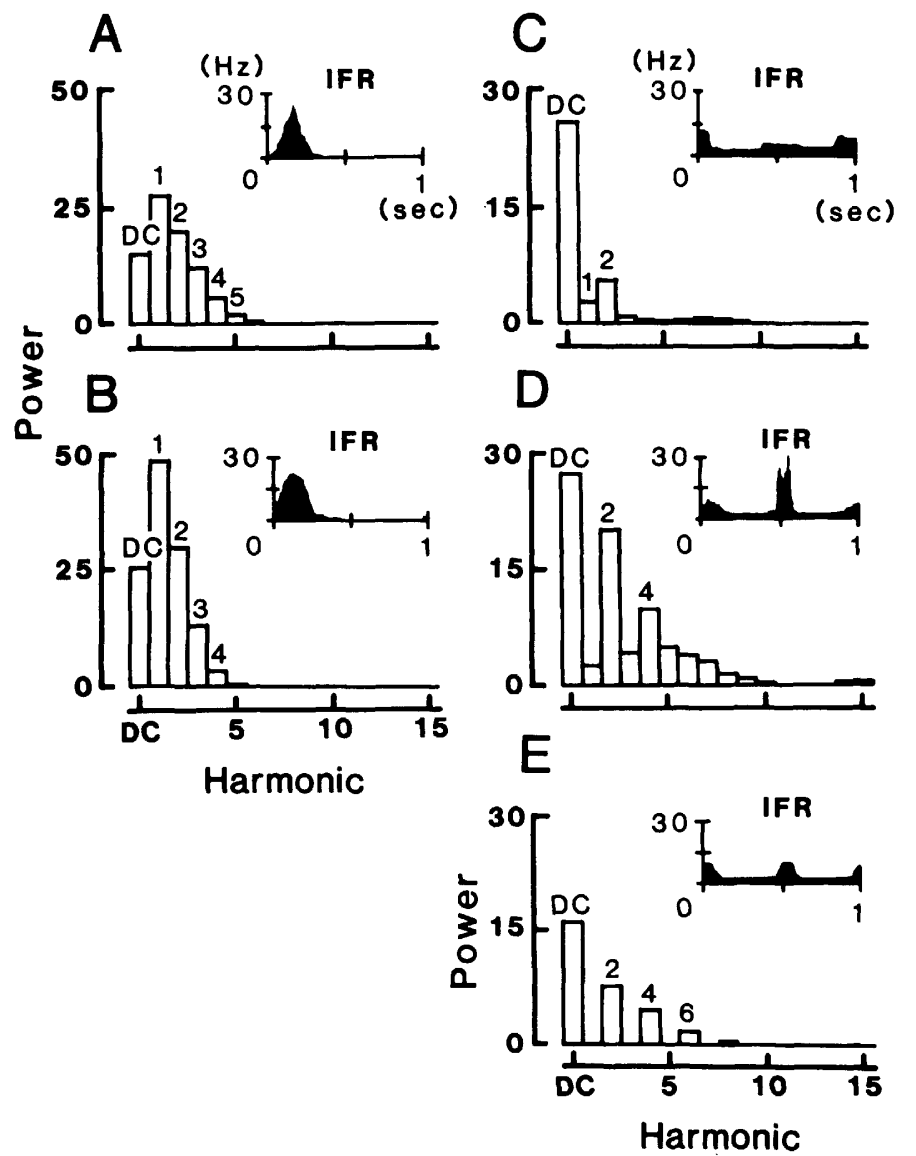

Fig. 3. Power spectra obtained from cycle time IFR of three types of mechanoreceptor afferent units to sinusoidal indentation of $1 \mathrm{~Hz}$. A: Ft I unit (the unit of Fig. 2A), B: Ft II unit (the unit of Fig. 2B), and C (the unit of Fig. 2C), to E: RA type I units. DC, $1,2,3, \cdots$ indicate direct current component (DC), fundamental wave, the 2nd, 3rd, and higher order harmonics. Ordinates represent powers of these components. Insets: cycle time IFRs to which FFT was applied. Abscissa indicates time (s).

much smaller in absolute value than the power of the fundamental wave of the SA units. The pattern of the power spectrum depended on the cycle time IFR. When an RA unit produced the largest IFR at a positive-going zero-crossing time point, the power of the fundamental wave was the second largest (Fig. 3C). When the IFR was not smaller at the negative-going zero-crossing time point than at the positive-going zero-crossing time point, the 4th harmonic became the second largest. In some IFRs, the power of harmonics of high orders was significantly large - that is, over $10 \%$ of the power of the 2 nd component (e.g., the 7 th harmonic in Fig. $3 \mathrm{C}$ and the 6 th harmonic in Fig. 3E). The power of the DC component was large in both the SA 

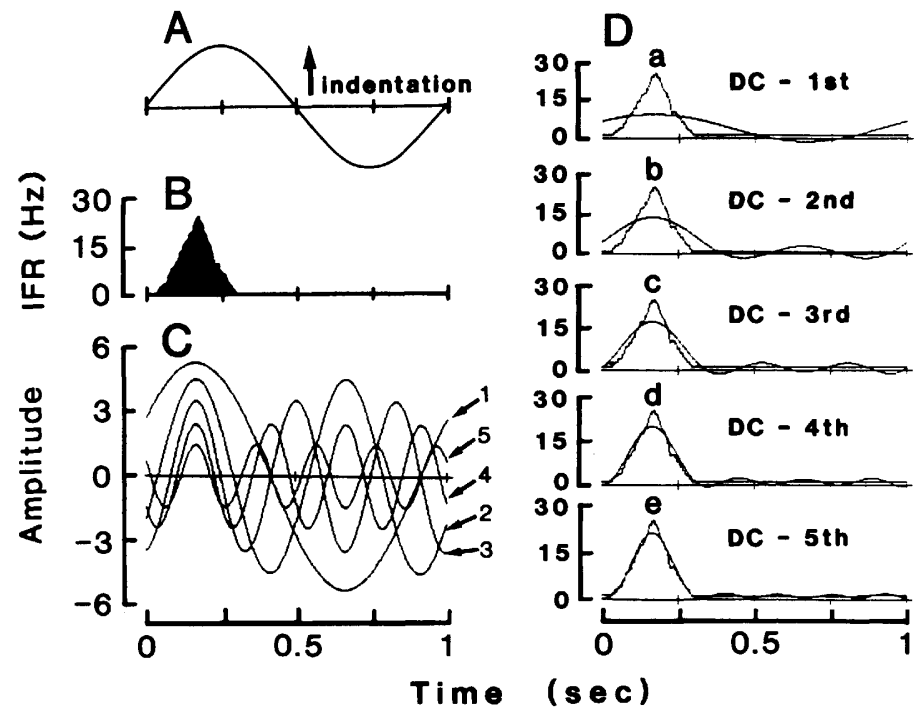

Fig. 4. Reconstruction of cycle time IFR of Ft I unit (the unit of 1A) by using DC component, fundamental wave and several harmonics in the power spectrum. A: sinusoidal stimulation of $1 \mathrm{~Hz}$. B: cycle time IFR. C: superimposed waveforms of the fundamental wave and harmonics through the 5th order. Magnitudes of these harmonics are given by a square root of the power. Numerals indicate the order of the harmonics. Da-De: original IFR and reconstructed response by adding the fundamental wave and the other 4 large harmonics to the DC component, with an appropriate phase shift.

and RA units, particularly in the latter, thereby suggesting large deviations of IFR from the sinusoidal waves.

The phase of the fundamental wave was advanced by $20-40^{\circ}$ relative to the sinusoidal stimulation in the Ft I units (Fig. 4C), and by $35-55^{\circ}$ in Ft II units. In both the Ft I and Ft II units, the positive peaks of the fundamental wave and higher harmonics were found at the same time interval from the positive-going zerocrossing time point (Fig. 4C), as that for the maximal peak of the instantaneous frequency response.

In the RA type I units, on the other hand, the positive peaks of the 2nd harmonic were found at the two zero-crossing time points of the sinusoidal stimulation. Those of even-order higher harmonics were also found near both the positive-going and negative-going zero-crossing time point. The phases of the evenorder harmonics were advanced by ca. $90^{\circ}$, in comparison with the stimulus wave (Fig. 5Ab, Bb, Cb). The phases of odd-order harmonics, including the fundamental wave, showed either advance (Fig. 5Ab) or lag (Fig. 5Bb) relative to the even-order harmonics, depending on the pattern of the cycle time IFR. They advanced by $90^{\circ}$ ahead of the stimulus wave when the IFR was the largest at the positive-going zero- 


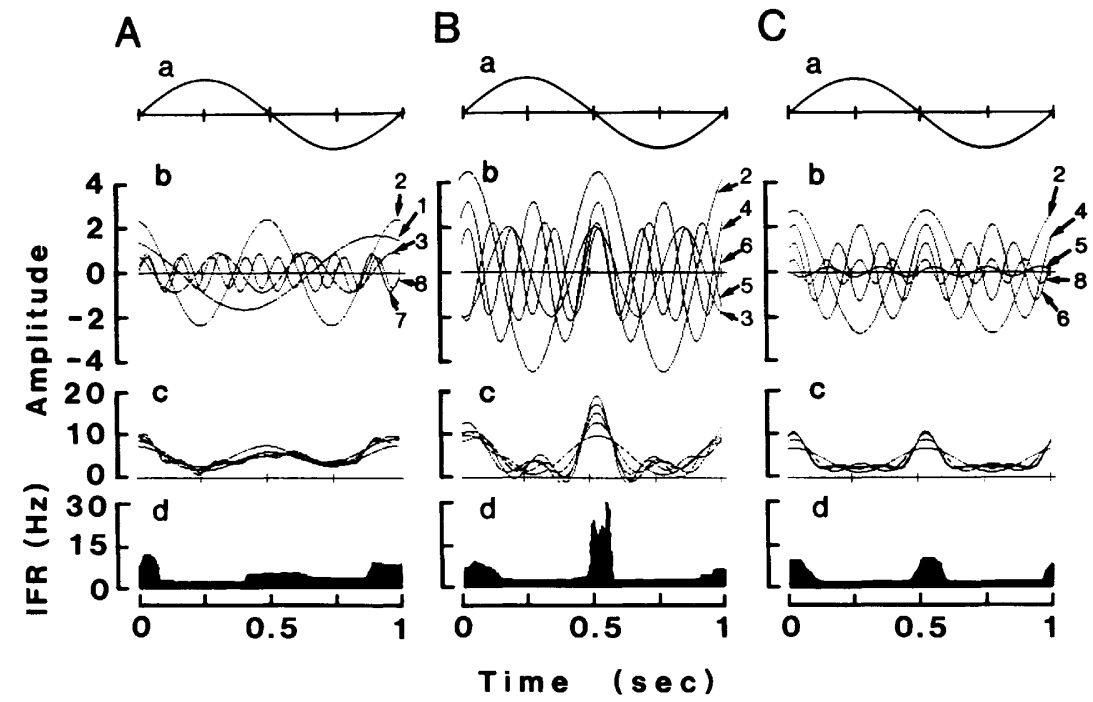

Fig. 5. Reconstruction of cycle time IFR of three RA type I units by the same procedure as in Fig. 3. a: sinusoidal stimulation of $1 \mathrm{~Hz}$ and $400 \mu \mathrm{m}$ in amplitude. b: superimposed waveforms of 5 components used to reconstruct IFR, except for DC. Numerals indicate the order of the harmonic. c: reconstructed IFR. d: original cycle time IFR.

crossing time point, while they lagged by ca. $90^{\circ}$ behind it when the IFR was the largest at the negative-going zero-crossing time point.

Reconstruction of original IFR using DC and major harmonics in the power spectrum

To clarify how many components of the power spectrum are needed to construct the IFR, we reconstructed IFR by adding several major components with appropriate phase shifts.

The reconstructed IFR of the Ft I units are shown in Fig. 4D. Since in SA units, the power of the fundamental wave is the largest and the power of the higher order harmonics decreases as the order is advanced, reconstruction was done by adding the fundamental wave to the DC component and the result was compared with the measured IFR. Then, the 2 nd harmonics was added and the result was reexamined. The same procedure was repeated until a good agreement was obtained (Fig. 4Da-e). As the number of added components increased, the reconstructed IFR gradually resembled the original one. Accumulation of harmonics from the 0 th order (DC component) to the 5th harmonic (Fig. 4De) resulted in a waveform which showed good agreement to the original IFR. The IFRs of the Ft II units were also reconstructed by using the largest five harmonics (fundamental wave to the 5 th harmonic) and the DC component, as in the case of the Ft I units.

In one of the RA type I units, the original IFR could be approximately 
reconstructed by summing the DC component and all the 5 major harmonics, e.g., 2nd, 4th, 5th, 3rd, and 6th harmonics (Fig. 5Bc). But the four largest harmonics, in addition to the DC components, were sufficient for the other two RA type I units: 2nd, 1st, 3rd, and 7th harmonics for the unit of Fig. 5A, and the 2nd, 4th, 6th, and 8th harmonics for the unit of Fig. 5C. Addition of other harmonic components did not significantly alter the waveform.

\section{Relation between power spectra and stimulus parameters}

$S A$ units. The pattern of IFR was independent of the amplitude or frequency of sinusoidal stimulation, except at low frequencies or large amplitudes. In both $\mathrm{Ft} \mathrm{I}$ and Ft II units, the power of the fundamental wave was the largest with every stimulus condition, and was followed by the 2 nd to the 5 th harmonics in a decreasing order of power. The power of the 1 st and 2 nd components exceeded $10 \%$ of the maximum power, across the entire range examined, both for indentation and frequency (Fig. 6). The power of the 3rd component exceeded $10 \%$ of the maximum power across the entire range examined, for only indentation. The magnitude of these major components of the power spectrum increased with increased amplitudes and frequencies of sinusoidal stimulation (Fig. 6A and C), but
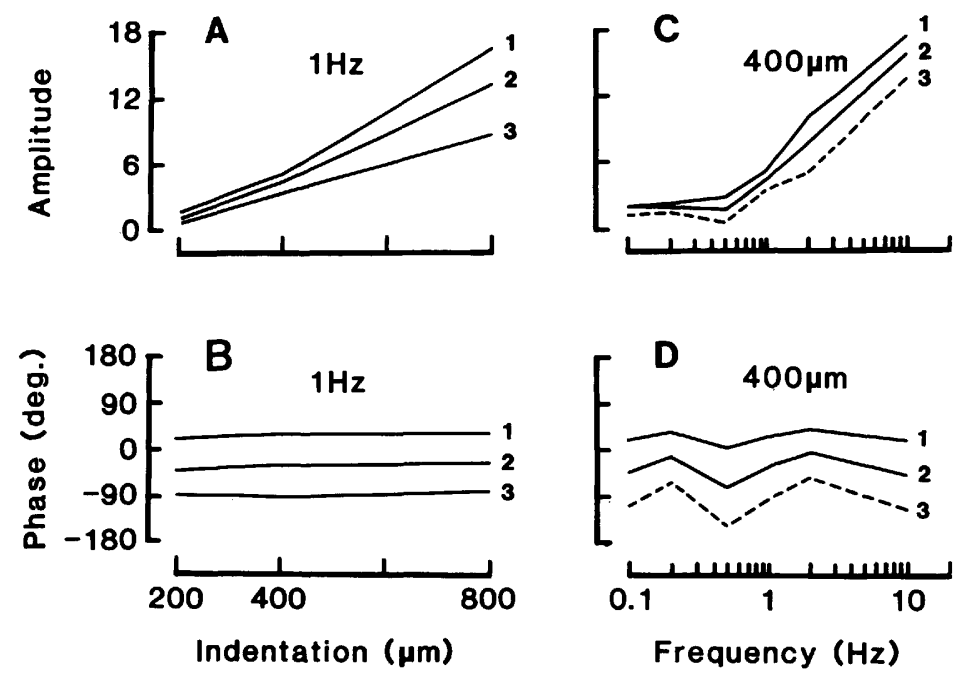

Fig. 6. Relation between the amplitude and phase of the 3 major harmonics to the amplitude of $1 \mathrm{~Hz}$ sinusoidal indentation in $\mathrm{Ft} \mathrm{I}$ unit (A and $\mathrm{B})$, and to the frequency of sinusoidal indentation $(400 \mu \mathrm{m}$ in amplitude) $(C$ and $D)$. Solid lines indicate the harmonics whose powers were $10 \%$ or larger of the maximum power in all spectra of IFRs obtained in both $1 \mathrm{~Hz}$ and $400 \mu \mathrm{m}$ amplitude series of stimulations. Broken lines indicate the harmonics whose powers were $10 \%$ or larger of the maximum power in the spectra of IFRs obtained in one of the two series of stimulations but smaller than $10 \%$ of the maximum power in a few spectra of IFRs in the other series of stimulations. Numerals indicate the order of the harmonics. 

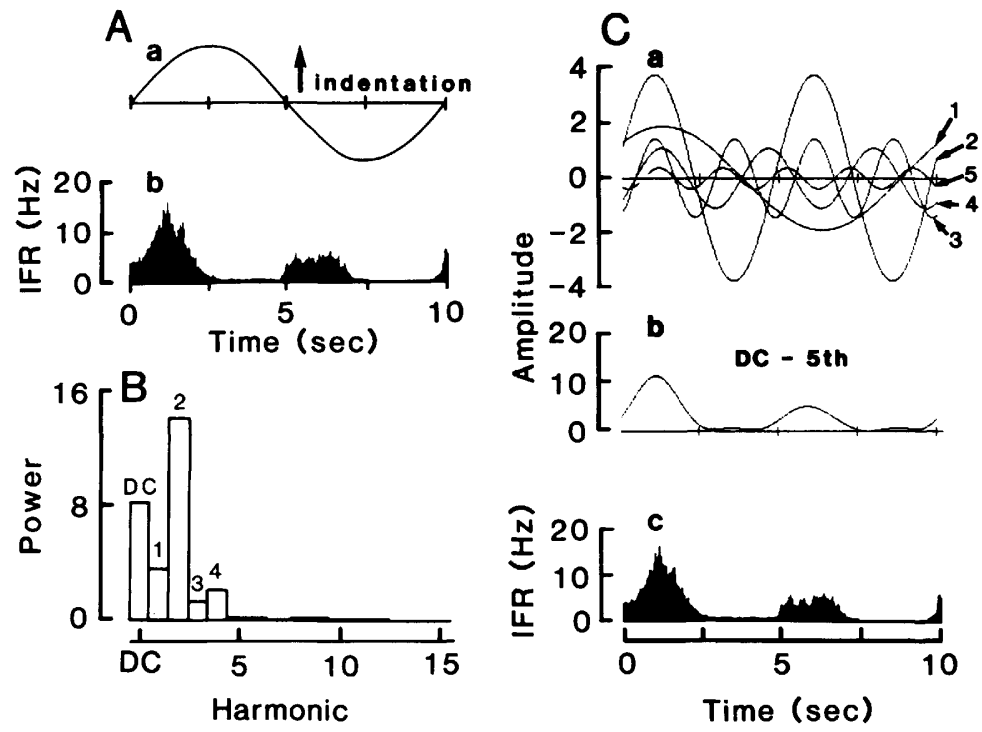

Fig. 7. Characteristics of cycle time IFR with two peaks in the Ft II unit seen under certain stimulus conditions. Aa: stimulus waveform of $0.1 \mathrm{~Hz}$ and $200 \mu \mathrm{m}$. Ab: cycle time IFR. B: relative power spectrum obtained from the cycle time IFR shown in $\mathrm{Ab}$. Ca: superimposed waveform of the harmonics. $\mathrm{Cb}$ : IFR reconstructed by summing the DC component, the fundamental wave and other harmonics of large power spectrum up to the 5th harmonics, while taking the phase of these harmonics into consideration. Cc: original cycle time IFR is the same as the one in Ab. DC and numerals in $\mathrm{B}$ and $\mathrm{Ca}$ indicate the order of harmonics.

the phases did not change significantly (Fig. 6B and D).

Among both types of SA units, five of the six Ft I and one of the three Ft II units showed two peaks in the cycle time IFR at a low frequency range $(0.1-0.5 \mathrm{~Hz})$ and mostly at maximal amplitudes of sinusoidal indentation $(800 \mu \mathrm{m}$ in four of the five Ft I units; $200 \mu \mathrm{m}$ in one Ft II unit) (Fig. 7Ab). This IFR pattern is similar to that of the RA type I units. However, a close examination showed that the two peaks occurred later than the two zero-crossing time points. Spectral analysis revealed that the power of the 2 nd harmonic was the largest (Fig. 7B), but that the phases of all the components in the spectrum, including the 2 nd harmonic, were almost the same as those of the SA units, with the IFR having a single peak in the case of other stimulus conditions (Fig. 7Ca).

$R A$ type I units. Increased amplitudes of sinusoidal stimulation remarkably altered the pattern of the cycle time IFRs. To a small amplitude $(200 \mu \mathrm{m})$, most of the IFR showed only a single peak at the positive-going zero-crossing time point. To a larger amplitude, two peaks appeared in the IFR, each at one of the two zerocrossing time points. Three patterns of the IFR with two peaks were also recognized (Fig. 3C-E), and these patterns did not change with alteration in the stimulus 
parameters. However, at a stimulation of the largest amplitude $(800 \mu \mathrm{m})$ and a frequency of $10 \mathrm{~Hz}$ or higher, the IFR showed only a single peak at one of the two zero-crossing time points.

Accordingly, the power spectrum of the RA type I units changed with varying amplitudes and frequencies of sinusoidal stimulation. In Fig. 8, powers of the 3 major components are plotted against stimulus parameters. The power of all these 3 components was large across the entire range of frequency, but only that of the 2nd component was large across the entire range of indentation. Of power spectra of the IFR with a single peak, the fundamental wave had the largest power. The power of the second harmonic was as large as that of the fundamental wave. When the IFR showed two peaks, the 2nd harmonic had the largest power (Fig. 8A and C). As shown in Fig. 5A-C, different patterns of the IFR with two peaks showed different power spectra. Even a slight change in the IFR affected the magnitude of some of the harmonics; e.g., when the peak of the IFR became sharper, the magnitude of the odd-order harmonics (e.g., 1st and 3rd components in Fig. 8A, C) became larger.

The phase of each component in the spectrum was found to advance by ca. $90^{\circ}$, on the average, and showed some variations with changes in stimulus parameters. Phases of the fundamental wave, 2nd, and other higher harmonics were 0 or $90^{\circ}$ to the stimulus of a low amplitude $(200 \mu \mathrm{m})$, which produced the IFR with a single peak. The phase advanced by $90-180^{\circ}$ in response to stimuli of larger amplitudes,
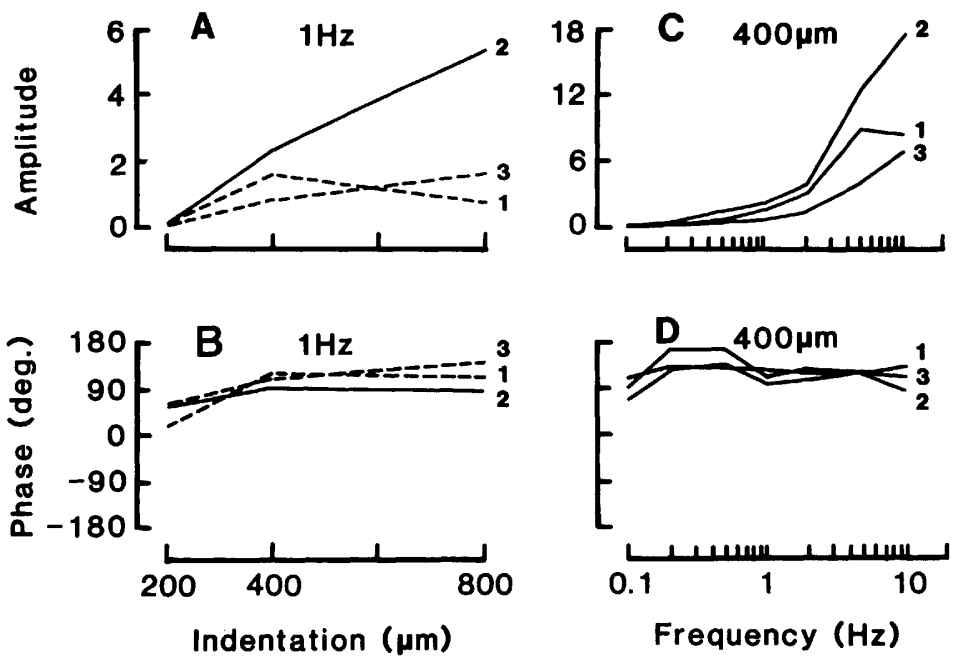

Fig. 8. Relation between characteristics of the 3 major harmonics and those of sinusoidal indentation in RA type I unit. The magnitude and phase of the 3 major harmonics, including fundamental wave are plotted against the amplitude of sinusoidal indentation at $1 \mathrm{~Hz}$ in $\mathrm{A}$ and $\mathrm{B}$. The magnitude and the phase of these harmonics are plotted against the frequency of sinusoidal indentation with $400 \mu \mathrm{m}$ in amplitude in C and D. For explanations of solid and broken lines, see the legend of Fig. 6. Numerals indicate the order of harmonics. 
which evoked two peaks in IFR (Fig. 8B). Stimulations of a large amplitude $(800 \mu \mathrm{m})$ and high frequency $(10 \mathrm{~Hz}$ or higher) produced IFRs with a single peak. The phase of the fundamental wave of this IFR was at either 0 or $180^{\circ}$, according to the pattern of the cycle time IFR.

\section{Adaptation and power spectrum of the IFR}

Some mechanoreceptor afferent units showed a marked decrease in spike discharges with advanced stimulus cycles (Fig. 2). In such units, different cycle time IFRs and power spectra were obtained at three different sections of 31 successive trials.

IFR. In Ft I units, the amplitude of IFRs decreased by $50 \%$ in the second section, relative to the first section, and the shape of the curve became sharper (Fig. 9Ab). Such a tendency was more marked in the third section (Fig. 9Ac). Despite these changes, the peak time of the IFRs did not shift throughout the three sections. It is suggested that both the accommodation and adaptation of the units increased as the stimulus cycle advanced.
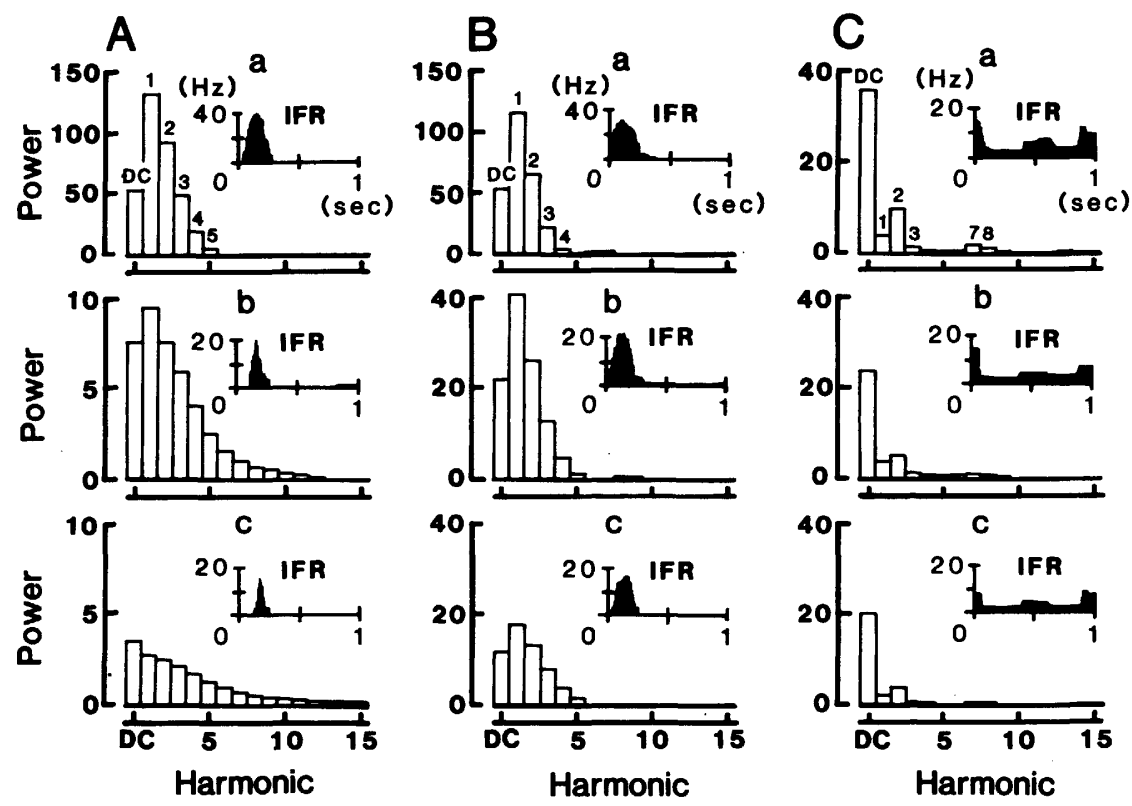

Fig. 9. Power spectrum obtained from cycle time IFR of three different types of units at three different sections of 31 successive sinusoidal indentations at $1 \mathrm{~Hz}$. A: Ft I unit $(400 \mu \mathrm{m}), \mathrm{B}: \mathrm{Ft}$ II unit $(100 \mu \mathrm{m})$, and C: RA type I unit $(400 \mu \mathrm{m})$. a: first section (2nd to 11 th trials). b: second section (12th to 21 st trials). c: third section (22nd to 31 st trials). DC, $1,2,3, \cdots$ indicate DC component, fundamental wave, the 2 nd, $3 \mathrm{rd}$, and higher order harmonics of the spectrum, respectively. Ordinates represent powers of the components. Insets: cycle time IFRs to which FFT was applied. Abscissa indicates time (s). 
In Ft II and RA type I units, on the other hand, magnitudes of cycle time IFRs decreased gradually and the maximum magnitude decreased by $50 \%$, only in the 3rd section (Fig. 9Bc and Cc). The shape of the IFR of Ft II units became sharper in a later section. The peak time of the IFRs in either type was not affected by repeated stimulations and was found at the same time point across the three sections.

Power spectrum. In Ft I units, the fundamental wave was the largest in all the three sections. In the first section, the 2 nd to 5 th harmonics were significant, but in later sections much higher harmonics, e.g., the 6th and 7th, became significantly larger in contrast to a decrease in the power magnitude of the first 5 harmonics and the DC component (Fig. 9Ab and Ac).

In Ft II units, on the other hand, the magnitude of all the components, including the DC component, became smaller in later sections and the magnitude of higher components, such as 3rd and 5th harmonics, became relatively larger (Fig. $9 \mathrm{Bb}$ and $\mathrm{Bc}$ ). Nevertheless, the pattern of the power spectrum was essentially the same throughout the three sections.

In the RA type I unit shown in Fig. 9C, the DC component, fundamental wave, and 2 nd harmonic were the three major components in the power spectrum across the three sections, and the higher harmonics were not significant in magnitude, in all three sections. The second harmonic was the most prominent in the first section, but in a later section, the magnitude decreased and was comparable to that of the fundamental wave. No significant changes in the power spectrum were evident over the three sections in the remaining RA type I units, with other cycle time IFRs.

\section{DISCUSSION}

Spectrum analysis, a technique of linear system analysis, has been used to study the dynamic responses of physical systems, since these systems show a linear performance. Use is often made of linear system analysis to study biological systems, including the nervous system (FrENCH et al., 1972; SAYERS, 1973; XerRi et al., 1987).

To quantitatively examine the dynamic response of cutaneous mechanoreceptor afferent units in frogs, we carried out a spectral analysis of their responses to sinusoidal stimulus. We applied FFT to the average cycle time IFRs of two types of the SA units (Ft I and Ft II units), and one type of the RA units (RA type I units). Cycle time IFRs are a kind of cross-correlation of the responses with the stimulus waveform (LEE, 1960; SATO et al., 1962), so that they are assumed to be noise-free responses evoked by stimulus.

To convert spike trains to an analogue waveform, there are at least two methods, i.e., the IFR and the method using counts of spikes per time bin. In the present study, we used IFR, a series of the reciprocals of ISIs assigned at the time periods of corresponding ISI, since neural information is considered to reside in variation of length of ISIs (BULLOCK, 1967). However, IFR gives an analogue wave with fewer peaks in a cycle of stimulus than that by the other method, as seen in the 
case of RA type I units in Fig. 2C, so that the present results may give a smaller power of high order harmonics to such mechanoreceptors.

RA type I units produced time-locked discharges, in contrast to responses in SA units (Fig. 2). Since the time-locked discharge indicates that spikes discharge at the same time intervals after the onset of stimulus cycle and with repeated stimulation, even the time-locked discharge in response to a single cycle stimulus may represent the average response over several stimulations. In other words, timelocked discharges mean noise-free discharges, discharges the most pertinent to application of FFT. In the present study, we used the average response of RA type I units to 31 trials of stimulations, as in the case of SA units.

In preceding work (TANIGUCHI et al., 1984), we studied the coding of cutaneous mechanoreceptor afferent units by examining the phase of their first spikes, in relation to the cycle of the sinusoidal stimulus. These threshold phases were easily affected by adaptation of the afferent units. Depending on the stimulus cycle, the threshold phase of these units may change.

In the present study, we used spectrum analysis by applying FFT to IFR of cutaneous mechanoreceptor afferent units. Since the main component of the power spectra was related to the peak of IFRs, which was stable in relation to the stimulus cycle during adaptation of the afferent units, the main feature of the coding mechanism of the afferent units was not affected by the adaptation.

Although the mechanoreceptor afferent units may encode two or more components of the mechanical stimulus, only the main component was directly obtained in the present study. Such complex features of the encoding mechanisms are simulated and discussed in a later section.

Spectrum analysis was applied to the units of responses which could be converted into IFRs. Since RA type II units discharged only one or two spikes in the cycle of sinusoidal stimulus waveform, they could not be used in the present analysis.

Possibility of encoding high orders of mechanical stimulation in SA units. In both types of SA units, the power of the fundamental wave was maximal and the phase advanced either by $20-40^{\circ}$ in Ft I units or $35-55^{\circ}$ in Ft II units. If a linear relation holds between the stimulus and response in SA units, only the fundamental frequency of stimulus sinusoidal wave should appear in the power spectrum of the response signals. In the present study, however, the IFR of the SA units to sinusoidal stimulation were a clipped version of the sine wave, thereby suggesting considerable harmonic distortion. As expected from the power spectrum of the halfrectified sinusoidal wave (BRACEWELL, 1978), the 2nd harmonic and 4th harmonic had considerable powers in the spectrum (Fig. 3AB, Fig. 4C). Similar findings have also been obtained from the cycle histogram of tactile spines to sinusoidal stimulation in the cockroach (FRENCH et al., 1972). It is assumed that the theoretical displacement detector gives rise to a train of impulse discharges frequencymodulated by sinusoidal stimulation, without any phase advance or lag, as seen in monkey SA units (TALBOT et al., 1968). The phase advance of the fundamental wave 
and the presence of significant powers of high harmonics suggest that both types of SA units encode higher orders of mechanical indentation, e.g., velocity as well as displacement.

Simulation of encoding both displacement and velocity components in SA units. We reconstructed IFRs of SA units by assuming that two types of SA units encode both displacement and velocity components of sinusoidal indentation. Our working hypothesis was 1) that the receptors of SA units encode both displacement (sine wave; wave I in Fig. 10B) and velocity (cosine wave; wave II in Fig. 10B), 2) that their responses are graded, proportionally related to the sum of these components (wave I + wave II), and 3) that the spike generation sites produce IFRs in proportion to the half-rectification of the receptor responses (wave III in Fig. 10B). We applied FFT to the IFRs of the hypothetical SA units and examined the relation between the phase advancement of the fundamental wave of these IFRs. We obtained the proportion of displacement components in responses at the receptor level (A1/(A1 + A2)) (Fig. 9A). The phase advance of $90^{\circ}$ indicates an ideal response ( $A_{1}=0$, only cosine wave) of a positive (indentation phase) velocity detector. With an increase in the percentage of the displacement response, the phase advance

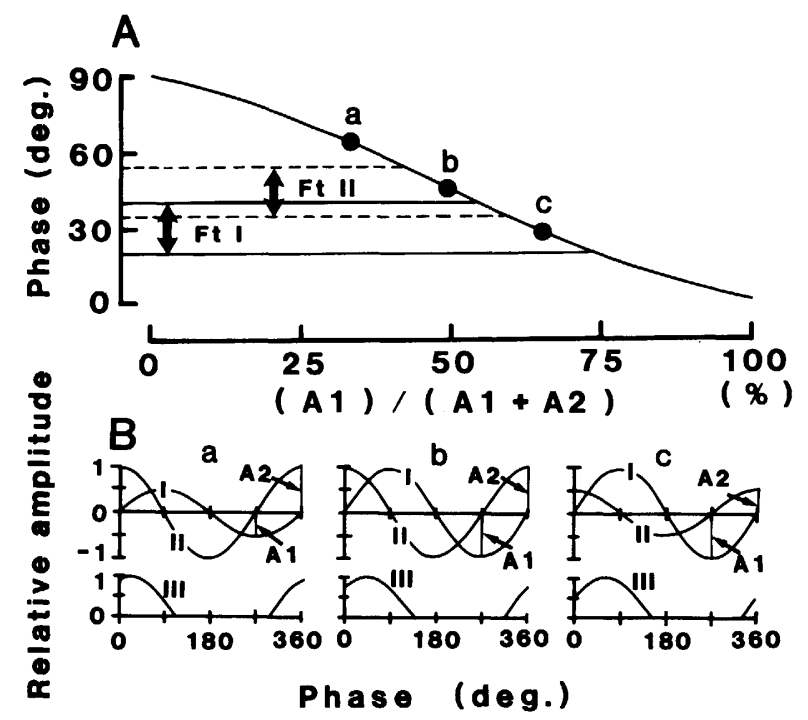

Fig. 10. Relation between the phase of the fundamental wave obtained from the hypothetical IFR (B, III) and the relative amount of displacement components in the IFR. The hypothetical IFR (B, III) was constructed by adding various magnitudes of sine wave (I) and cosine wave (II) and clipping the resultant wave below the zero level; where the sine wave (B, I) and cosine wave (B, II) represent the IFRs of the ideal displacement receptor and the ideal velocity receptor, respectively. The vertical axis shows the phase of the advance or lag of the fundamental wave, obtained by applying FFT to the hypothetical IFR, relative to the sine wave representing the stimulus waveform, as well. The horizontal axis indicates the percentage of the displacement response (I) to the complete response $(\mathrm{I}+\mathrm{II})$. 
gradually decreases to zero; e.g., when both the displacement and velocity responses evenly contribute to the whole response $\left(A_{1}=A_{2}, 50 \%\right)$, the peak phase advances by $45^{\circ}$. Finally, the null phase advance indicates the ideal displacement response $\left(A_{2}=\right.$ 0 , only sine wave).

By comparing the phase of the fundamental wave obtained from the IFR in our experiment with that of the simulated IFR in Fig. 9, we can estimate the amount of both the displacement and the velocity components of the IFR, in SA units. Since phases of the fundamental wave of the $\mathrm{Ft}$ I and $\mathrm{Ft}$ II units advanced by $20-40^{\circ}$ or $35-55^{\circ}$, respectively, it was assumed that both the Ft I and Ft II units encode the two components of the sinusoidal indentation with a relative preponderance of the displacement component.

The difference in the peak phase of the fundamental wave between the Ft I and Ft II units might be caused by a difference in the transduction mechanism and the physical nature of the tissue surrounding these receptors. Ft II units are found only in non-warty skin. Ft I units were recorded only from the warty skin in the present study, though they are present both in warty and non-warty skin (OGAWA et al., 1984). It was not clear from the present study whether phase differences between the two types of units can be attributed to differences in the physical nature between the two types of skin.

The present finding is compatible with previous results obtained from the responses to "ramp and plateau" stimulations. SA units produce phasic responses at the onset of the ramp indentation, with an increased IFR in response to a steeper slope of the ramp indentation (HoRCH and BURGESS, 1976). However, the present conclusion concerning performance of SA units is incompatible with previous results obtained using the threshold response phase method: we reported that the $\mathrm{Ft}$ II units performed like an ideal displacement detector at a small range of frequency of sine wave indentation, but that the Ft I units were less satisfactory in functioning as displacement detectors (TANIGUCHI et al., 1984).

Coding mechanism of sinusoidal wave in RA type I units. On the basis of the responses to "ramp and plateau" indentations, it has been assumed that the RA type I units encode the velocity component of mechanical stimulation. In our previous study using the threshold response phase method, we found that performance of the RA I units did not fit that of the theoretical velocity detectors, except for the performance at small skin indentations with a low frequency component (TANIGUCHI et al., 1984).

The IFRs of the RA type I units to a $1 \mathrm{~Hz}$ sinusoidal stimulation showed two peaks, with one each of the zero-crossing (Fig. 2C). The waveform is similar to that of the fully-rectified stimulus waveform. It is evident that RA type I units encode sinusoidal stimulation, non-linearly. This type of non-linearity has also been found in the response of vestibular nucleus neurons of cats (XERRI et al., 1987). Spectral analysis revealed that the 2 nd harmonic was the largest in the power spectrum of the IFRs (Fig. 3C, D, and E), apart from the DC component. The IFR pattern of the RA type I units is determined mainly by the relative power of the 2 nd harmonic. 
The RA type I units gave rise to sinusoidal responses with the angular velocity being twice as large as that of the stimulus. Since the square of a trigonometric function with an angular velocity of $\omega$ is expressed by the function with the angular velocity of $2 \omega$, RA type I units produce so-called "square-law responses" (GRAHAM and MCRUER, 1961).

The 2 nd harmonic showed its positive peaks near the two zero-crossing time points, positive-going and negative-going, at which the sinusoidal stimulation has its highest positive and negative velocity. The phase advance of the 2 nd harmonic to the sinusoidal stimulation was constant at ca. $90^{\circ}$, with varying amplitudes and frequencies of sinusoidal indentation (Fig. 8B and D). The present finding suggests that the RA type I units encode an absolute velocity of sinusoidal stimulation.

Physiological basis of square-law responses in $R A$ units. It has been generally accepted that most of the RA units have receptors of lamellated corpuscles acting as a mechanical high pass filter to mechanical indentations, so that they encode only the dynamic phase of the displacement (LoEwEnSteIn and SKalaK, 1966; LOEWENSTEIN, 1971; IGGo and ANDRES, 1982).

Thus, when a mechanical stimulation is applied to the lamellated corpuscle, it produces compression to the nerve terminal twice, at the onset and offset of the stimulus. This occurs because the volume of the space inside the lamella is decreased twice-(1) by active compression of the lamella at the active phase of indentation, and (2) by an overshoot of the passive elastic return of the lamella to the resting state at the withdrawal phase. Lamellated corpuscles are also present in the frog skin (DURING and SEILER, 1974). If the RA type I units do innervate such corpuscles, it is reasonable to assume that the lamella is responsible for the "square-law responses."

We express our gratitude to Dr. J. Kashiwagi and Mr. M. Sakata for pertinent advice on the data analysis and to M. Ohara for comments on the manuscript. Mr. Y. Yamashita participated in a part of the present experiment.

\section{REFERENCES}

Bendat, J. S. and Piersol, A. G. (1980) Engineering Application Correlation and Analysis, John Wiley \& Sons, Inc., New York, pp. 264-291.

Bracewell, R. N. (1978) The Fourier Transform and Its Applications, 2nd ed., McGrawHill, New York, pp. 206-207, pp. 396-397.

Bullock, T. H. (1967) Signals and neural coding. In: The Neurosciences. A Study Program, ed. by Quarton, G. C., Melnechuk, T., and Schmitt, F. O., The Rockefeller University Press, New York, pp. 347-352.

Burgess, R. and Perl, E. (1973) Cutaneous mechanoreceptors and nociceptors. In: Handbook of Sensory Physiology, ed. by IGGo, A., Springer-Verlag, Berlin, Vol. 2, pp. 29-78.

Charnock, D. M. and Manenica, I. (1978) Spectral analysis of R-R intervals under different work conditions. Ergonomics, 21: 103-108.

COOLEY, G. W. and TukEY, J. W. (1965) Algorithm for the machine calculation of complex Fourier series. Math. Comput., 19: 297-301. 
Cremers, P. F. L. J. M. (1972) Method for analyzing the spike response to sinusoidal stimulation. Electroencephalogr. Clin. Neurophysiol., 32: 448-452.

During, M. von and SeILER, W. (1974) The fine structure of lamellated receptors in the skin of Rana esculenta. Z. Anat. Entwickl. Geach., 144: 165-172.

French, A. S., Holden, A. V., and Stein, R. B. (1972) The estimation of the frequency response function of a mechanoreceptor. Kybernetik, 11: 15-23.

Gibson, J. M., BeITER, R. E., and WeLKeR, W. (1975) Diversity of coding profiles of mechanoreceptors in glabrous skin of kittens. Brain Res., 86: 181-203.

Graham, D. and McRuer, D. (1961) Analysis of Nonlinear Control Systems, John Wiley \& Sons, Inc., New York, pp. 28-29.

Horch, K. W. and Burgess, P. R. (1976) Effect of activation and adaptation on the sensitivity of slowly adapting cutaneous mechanoreceptors. Brain Res., 98: 109-118.

IgGo, A. (1974) Cutaneous receptor. In: The Peripheral Nervous System, ed. by HubBard, J. I., Plenum Press, New York, pp. 347-404.

IgGo, A. and Andres, K. H. (1982) Morphology of cutaneous receptors. Annu. Rev. Neurosci., 5: 1-31.

LeE, Y. W. (1960) Statistical Theory of Communication, John Wiley \& Sons, Inc., New York, pp. 155-176.

Loewenstein, W. R. (1971) Mechano-electric transduction in the Pacinian corpuscle. Initiation of sensory impulses in mechanoreceptors. In: Handbook of Sensory Physiology, ed. by Loewenstein, W. R., Springer-Verlag, Berlin, Vol. 1, pp. 269-290.

Loewenstein, W. R. and Skalak, R. (1966) Mechanical transmission in a Pacinian corpuscle. An analysis and a theory. J. Physiol. (Lond.), 182: 346-378.

LUCZAK, H. and LAURIG, W. (1973) An analysis of heart rate variability. Ergonomics, 16: 85-97.

Ogawa, H., Morimoto, K., and Yamashita, Y. (1981) Physiological characteristics of low threshold mechanoreceptor afferent units innervating frog skin. Q. J. Exp. Physiol., 66: $105-116$.

Ogawa, H., Yamashita, Y., Nomura, T., and Taniguchi, K. (1984) Discharge patterns of the slowly adapting mechanoreceptor afferent units innervating the non-warty skin of the frog. Jpn. J. Physiol., 34: 255-267.

Sato, K., Honda, N., Mimura, K., Ozaki, T., Masuya, S., Teramoto, S., and Kitajima, K. (1962) A simplified method for crosscorrelation analysis. Electroencephalogr. Clin. Neurophysiol., 14: 767-768.

SAYERS, B. McA. (1973) Analysis of heart rate variability. Ergonomics, 16: 17-32.

Talbot, W. H., Darian-Smith, I., Kornhuber, H. H., and Mountcastle, V. B. (1968) The sense of flutter-vibration: Comparison of human capacity with response patterns of mechanoreceptive afferents from the monkey hand. J. Neurophysiol., 31: 301-334.

Taniguchi, K. and OGawa, H. (1986) Spectral analysis of instantaneous frequency response to sinusoidal stimulation in mechanoreceptor afferent units of frog skin. $J$. Physiol. Soc. Jpn., 48: 313.

TaniguChI, K., Yamashita, Y., and Ogawa, H. (1984) Threshold response phase to sinusoidal stimulation of frog cutaneous mechanoreceptor afferent units. Jpn. J. Physiol., 34: 1065-1075.

Xerri, C., Barthélémy, J., Harlay, F., Borel, L., and Lacour, M. (1987) Neuronal coding of linear motion in the vestibular nuclei of the alert cat. I. Response characteristics to vertical otolith stimulation. Exp. Brain Res., 65: 596-581. 